\title{
CORTICOSPINAL DYSGENESIS AND CONGENITAL HEMIPLEGIA
}

A diffusion tensor imaging technique was compared with conventional MRI to measure and quantify corticospinal dysgenesis in 12 patients with congenital hemiplegia and 12 matched control subjects, in a study at Universite Catholique de Louvain, Brussels, Belgium. A symmetry index computed between the area of the contralateral and ipsilateral corticospinal tracts was similar for the two methods, but the diffusion tensor imaging indexes were significantly smaller. This suggests that the use of the conventional MRI measurement of the cross-sectional area of cerebral peduncles on T1 MRI might lead to an underestimate of cortical dysgenesis. Hand-movement deficits, particularly precision grasping, and stereognosis were examined and correlated with the neuroimaging findings. The symmetry index computed from MRI peduncle measurements correlated solely with deficits in stereognosis, while the diffusion tensor imaging index correlated with stereognosis, digital and manual dexterities, and a measure of manual ability in daily life activities. (Bleyenheuft Y, Grandin CB, Cosnard G, Olivier E, Thonnard J-L. Corticospinal dysgenesis and upperlimb deficits in congenital hemiplegia: a diffusion tensor imaging study. Pediatrics Dec 2007;120(6):e1502-e1511). (Respond: Jean-Louis Thonnard PhD, Universite Catholique de Louvain, Unite de Readaptation, Ave Mounier 53, 1200 Bruxelles, Belgium).

COMMENT. Diffusion tensor imaging symmetry index may prove useful in predicting motor and sensory deficits in children with congenital cerebral dysgenesis.

\section{SEIZURE DISORDERS}

\section{SURGERY FOR INTRACTABLE TEMPORAL LOBE EPILEPSY IN YOUNG CHILDREN}

The results of temporal resection for medically intractable epilepsy in 20 children less than age 5 years with at least 2 years follow-up are reported from Miami Children's Hospital, Florida. The mean age at surgery was 26 months, and the mean age at seizure onset was 12 months. Seizures were typical psychomotor in 4 patients, with staring and oral or gestural automatisms; and psychomotor plus in 7, with aura and frightened appearance, staring, decreased responsiveness followed by movements that were contraversive, lateralized tonic or clonic, and asymmetric tonic posturing. Motor symptoms were prominent in 3 , with tonic asymmetric posturing followed by circling and vegetative signs. Clusters of epileptic spasms occurred in 6, mainly clonic. Interictal EEGs showed lateralizing abnormalities in 15 that were concordant, and ictal EEGs were lateralizing and concordant in 18 and nonlateralizing in 2. Brain MRI revealed localizing pathology in 16, and ictal SPECT was concordant in 4/8 cases. Invasive EEG recording was performed in 6 children to delineate the epileptogenic zone and map language cortex. Electrocorticography was performed in the remaining 14 cases. At mean follow-up of 5.5 years following surgery, $65 \%$ were seizure-free and $15 \%$ had $>90 \%$ seizure reduction. The etiologic pathology was a tumor in 8 cases, benign developmental in 4 and malignant astrocytoma in 4. Focal cortical dysplasia was found in 6 cases, one with neurofibromatosis. Hippocampal sclerosis was identified in 4 . Other pathologies included encephalitis, prior hypoxic-ischemic event, tuberous sclerosis, and 